\title{
Smart Nanotheranostics Responsive to Pathological Stimuli
}

\author{
Alessandro Parodi ${ }^{1 *}$, Magdalena Rudzinska $^{1}$, Stefano Leporatti ${ }^{2}$, Yuri Anissimov ${ }^{1,3}$ and \\ Andrey A. Zamyatnin Jr. ${ }^{1,4 *}$ \\ ${ }^{1}$ Institute of Molecular Medicine, Sechenov First Moscow State Medical University, Moscow, Russia, ${ }^{2}$ CNR NANOTEC - \\ Istituto di Nanotecnologia, Polo di Nanotecnologia, Lecce, Italy, ${ }^{3}$ School of Environment and Sciences, Griffith University, \\ Gold Coast, QLD, Australia, ${ }^{4}$ Belozersky Institute of Physico-Chemical Biology, Lomonosov Moscow State University, \\ Moscow, Russia
}

\section{OPEN ACCESS}

Edited by:

Gabriele Candiani,

Politecnico di Milano, Italy

Reviewed by:

Enzo Terreno,

University of Turin, Italy

Melani Solomon,

University of Maryland, United States

Jing Lin,

Shenzhen University, China

*Correspondence:

Alessandro Parodi

aparodi.sechenovuniversity@

gmail.com

Andrey A. Zamyatnin Jr.

zamyat@belozersky.msu.ru

Specialty section:

This article was submitted to

Nanobiotechnology,

a section of the journal

Frontiers in Bioengineering and

Biotechnology

Received: 04 March 2020

Accepted: 29 April 2020

Published: 25 May 2020

Citation:

Parodi A, Rudzinska M, Leporatti S,

Anissimov $Y$ and Zamyatnin AA Jr (2020) Smart Nanotheranostics Responsive to Pathological Stimuli.

Front. Bioeng. Biotechnol. 8:503.

doi: 10.3389/fbioe.2020.00503
The development of nanotheranostics represents one of the most dynamic technological frontiers in the treatment of different pathological conditions. With the goal in mind to generate nanocarriers with both therapeutic and diagnostic properties, current research aims at implementing these technologies with multiple functions, including targeting, multimodal imaging, and synergistic therapies. The working mechanism of some nanotheranostics relies on physical, chemical, and biological triggers allowing for the activation of the therapeutic and/or the diagnostic properties only at the diseased site. In this review, we explored new advances in the development of smart nanotheranostics responsive to pathological stimuli, including altered $\mathrm{pH}$, oxidative stress, enzymatic expression, and reactive biological molecules with a deep focus on the material used in the field to generate the particles in the context of the analyzed disease.

Keywords: nanotheranostics, smart nanoparticles, pH-responsive theranostics, ROS-responsive theranostics, Enzyme-responsive theranostics

\section{INTRODUCTION}

Nanotheranostic development perhaps represents the highest level of technological advance in the nanomedicine field, and it aspires to combine in the same delivery platform therapeutic and diagnostic properties (Wong et al., 2020). Nanotheranostics are nanoparticles designed to provide real-time information about drug biodistribution, release, and targeted treatment in vivo, representing one of the last frontiers in personalized medicine (Jo et al., 2016). Nanotheranostics are usually generated through complex synthetic protocols (Silva et al., 2019) necessary to transfer multiple functions to the same delivery platform. For this reason, in several cases, nanotheranostics' targeting simply relies on particle passive accumulation in the diseased tissue via enhanced permeability and retention effect (EPR) usually achieved with biological coating (i.e., albumin, peptides) or polyethylene glycol (PEG) surface modification.

However, beyond exploiting a specific targeting, nanotheranostic selectivity for the pathological area can derive from a specific "responsiveness" of the carriers to an external stimulus (Sneider et al., 2017). This "activation trigger" (e.g., near-infrared light) is usually remotely applied directly in the area of interest (Wang et al., 2017b) and has benefits of non-invasiveness. Typical diagnostic or therapeutic carriers are generally in the "on" state (Zhao et al., 2017), and their detection and payload release occur from the moment of their administration. On the other hand, the designing of carriers with "off-on" theranostic properties (Yu et al., 2018) can favor a personalized assessment of the amount of drug that effectively reaches the pathological site. For this reason, responsive nanotheranostics have the potentialities to open new avenues for optimizing and controlling the 
treatment dose and repetition (Fan et al., 2016; Yang et al., 2016b). In this scenario, it seems almost impossible to increase further the level of complexity of these technologies. To date, many theranostics can permit multimodal imaging and therapy for enhancing diagnostic accuracy and treatment efficacy (Dong et al., 2016). However, new trends in nanomedicine aim at imparting the carriers with responsiveness to the biological environment conditions. In other words, the physiological alterations that differentiate diseased from healthy tissue can serve as "triggers" to turn in vivo the nanotheranostics "on" (Ma et al., 2016). Also, these concepts showed the potential to improve current experimental protocols. For example, nanotheranostics that can enhance their detection signal in response to precise biological stimuli could mitigate the background noise issues related to the imaging of fluorescently modified carriers ( $\mathrm{Wu}$ et al., 2014).

The working mechanism of the stimuli-responsive carriers, often referred to as "smart" theranostics, usually depends on functional molecules or chemical linkers used to assemble the nanoparticles (Karimi et al., 2016). However, in some cases, it is the intimate structure of the particles that changes or responds to the environmental conditions, like in the case of polymers (Li et al., 2019a). In this review, we explored recent advances in the development of nanotheranostics that can respond to biological stimuli. These carriers were designed to provide their curative and/or diagnostic properties only in the pathological site, exploiting altered chemical and biological features of the diseased tissue. These concepts, as well as the field of nanomedicine, are traditionally applied to cancer disease because tumor tissue undergoes profound changes in cell metabolism, generating significant variations in local $\mathrm{pH}$ and oxidative stress. Sometimes the nanoparticles were designed to respond to more than one biological stimuli as well as in combination with physical stimuli that can be remotely administered. Also, tumor growth very often depends on the differential expression of enzymes that can be exploited as biological triggers as well (Xiao et al., 2018). However, recent evidence demonstrated that the concepts of biologically responsive nanotheranostic could be beneficial also for other pathological conditions, expanding the application of these technologies to a new whole portfolio of clinical conditions.

\section{PH-RESPONSIVE THERANOSTICS}

In the area of cancer therapy, $\mathrm{pH}$ represents an essential cue of differentiation compared to healthy tissue. Even though not all the regions of the tumor become significantly acidified, changes in cancer metabolism [i.e., the Warburg effect (Tekade and Sun, 2017; Shamsi et al., 2018)], can induce an overall average decrease of $0.2-0.4$ points, although values $<6$ were registered as well (Liu et al., 2014a). In this scenario, the tumor microenvironment $\mathrm{pH}$ can eventually represent a targetable characteristic. Also, after cell internalization, the nanoparticles are usually sequestered in the endolysosomal compartment, where the $\mathrm{pH}$ drops significantly below 5 (Wang et al., 2017a), making these organelles optimal targets for $\mathrm{pH}$-responsive technologies.
$\mathrm{pH}$-responsive properties can be coupled with the activation of different therapeutic mechanisms, including reactive oxygen species (ROS)-based cytostatic therapeutics, as well as different imaging modalities. In this context, sono-, photo-, and chemodynamic therapies are treatments in which the cytostatic properties rely on the overproduction of ROS. In sono- and photodynamic therapy (PDT), ROS can be generated upon an external stimulus. In contrast, chemodynamic therapy (CDT) depends just on the chemical properties of the carriers or the payload responsible for ROS generation, usually when interacting with the cellular $\mathrm{H}_{2} \mathrm{O}_{2}$ (Lin et al., 2018).

The $\mathrm{pH}$-responsiveness can derive from $\mathrm{pH}$-sensitive linkers used to stabilize the particles (Kanamala et al., 2016) or its payload in the carrier structure. An extensive overview of this topic can be found in Cao et al. (2019). Acotinyl linkers were intensely investigated in the field to impart $\mathrm{pH}$ responsiveness to the carriers. For example, Zhu et al. generated $\mathrm{pH}$-responsive theranostics incorporating gold nanoparticles in poly(amidoamine) dendrimers (Zhu et al., 2018). The gold nanoparticles represented an optimal contrast agent in computed tomography (CT) imaging. The dendrimers were modified on their surface with folic acid via EDC chemistry to provide the particles with high tumor targeting. Cis-aconitic anhydride $\mathrm{pH}-$ sensitive linkers were used to conjugate Doxorubicin (DOX) to the particles. Drug release was triggered by the acidic conditions (typical of the tumor microenvironment and the endosomal compartment) even though in vivo proof of the efficacy of this technology was not reported. Similarly, nanotheranostics were generated with bovine serum albumin (BSA) linked to the porphyrin photosensitizer pheophorbide-a via cis-aconityl $\mathrm{pH}$-sensitive linkers. Stable nanoparticles were generated by complexing this structure with graphene oxide via $\pi-\pi$ stacking and hydrophobic interactions (Battogtokh and Ko, 2016). The carriers were further modified with pegylated folate to impart the system with extended circulation properties and tumor targeting. Porphyrins are known for their therapeutic potential (PDT) and fluorescent properties, but their administration requires encapsulation since they are strongly hydrophobic (Yan et al., 2015). This complex facilitated dual therapy through PDT and photothermal therapy (PTT) due to the porphyrin payload and the graphene oxide, respectively, upon irradiation at $670 \mathrm{~nm}$. In vivo, compared with the free administered photosensitizer, the particle highly accumulated in the tumor tissue through EPR effect. Here they could release their payload due to the $\mathrm{pH}$ sensitive linkers both in the acidic tumor microenvironment and well as in the cell cytoplasm after internalization favored by the folate functionalization. The $\mathrm{pH}$-sensitive mechanism was fundamental to activate the theranostic properties of the porphyrin since this molecule is affected by aggregation-caused quenching (ACQ) effect when encapsulated.

On the other hand, different materials can be manipulated in the nanoscale and dissolve (releasing a payload) in acidic $\mathrm{pH}$. Calcium carbonate $\left(\mathrm{CaCO}_{3}\right)$ nanoparticles represent a wellinvestigated delivery platform (Idris et al., 2019; Vidallon et al., 2020 ) in this field. At physiological $\mathrm{pH}, \mathrm{CaCO}_{3}$ nanoparticles are stable, but under acid conditions, they degrade into $\mathrm{Ca}^{2+}$ and $\mathrm{CO}_{2}$, releasing whatever payload was previously 
loaded. Pegylated $\mathrm{CaCO}_{3}$ nanoparticles were synthesized by gas diffusion-reaction in combination with the photodynamic theranostic agent chlorin e6 (Ce6) (Dong et al., 2016). Like porphyrins, Ce6 is per se a theranostic because it can serve as a therapeutic tool for PDT while emitting a detectable fluorescent signal upon near-infrared light (NIR) irradiation (Liu et al., 2014b). The particles were also doped with $\mathrm{Mn}^{2+}$, to favor the photosensitizer precipitation with the $\mathrm{CaCO}_{3}$. $\mathrm{Mn}^{2+}$ also allowed for detecting particle degradation and payload release via magnetic resonance imaging (MRI), and it provided a mean for CDT increasing ROS levels via Fentonlike reaction with tissue $\mathrm{H}_{2} \mathrm{O}_{2}$. In this chemical reaction a transition metal (i.e., $\mathrm{Fe}, \mathrm{Al}, \mathrm{Mn}, \mathrm{Cu}, \mathrm{Zn}$ ) interacting with $\mathrm{H}_{2} \mathrm{O}_{2}$ is oxidized while catalyzing the formation of a hydroxyl radical and a hydroxide ion (Das et al., 2015). The system was characterized by a mesoporous structure compatible with the loading of conventional chemotherapeutics like DOX that, in acidic conditions, was released as well. The particles were also modified with PEG allowing for extravasation in the tumor microenvironment via EPR. Under the acidic conditions of cancer tissue, they could exert their theranostic properties demonstrating high cytostatic properties both in vitro and in vivo against a model of breast cancer, The authors demonstrated that chemotherapy (DOX), CDT $\left(\mathrm{Mn}^{2+}\right)$ and PDT (Ce6) could work synergistically against cancer cell growth (Dong et al., 2016) accomplishing multimodal therapy.

Another example of $\mathrm{pH}$-responsive structure was proposed by Xiao et al. (2019) that developed a theranostic platform named MCDION-Se able to provide at the same time chemodynamic and limotherapy while representing an optimal contrast agent for MRI application. The system was composed of a core of manganese carbonate-deposited iron oxide nanoparticles coated with negatively charged selenium nanoparticles coordinated to the core via polyethylenimine (PEI) (Figure 1). The manganese carbonate in the system could easily dissolve in slightly acidic conditions releasing $\mathrm{Mn}^{2+}$ for CDT and MRI while inhibiting ATP generation. On the other hand, the presence of the selenium enhanced the formation of $\mathrm{H}_{2} \mathrm{O}_{2}$, fueling the Fenton-like reaction catalyzed by the iron oxide nanoparticles to form ROS that further inhibited ATP synthesis. The combination of these elements was designed to breakdown in the tumor microenvironment and tumor cells after internalization, triggering different cascades of events affecting the energetic metabolism (limotherapy) while accelerating cell apoptosis via CDT. In vivo, the particles showed a higher circulation time and tumor accumulation than free $\mathrm{Mn}^{2+}$, probably via the EPR effect even though specific modifications to favor this phenomenon were not described. Manganese was also exploited by Liu et al. that designed PEG-coated $\mathrm{MnO}_{2}$ nanoparticles stabilized with BSA. The particles were loaded with the radiosensitizer hafnium and a prodrug form of cisplatin. The core of $\mathrm{MnO}_{2}$ could catalyze the conversion of tumor $\mathrm{H}_{2} \mathrm{O}_{2}$ to $\mathrm{O}_{2}$ to revert hypoxic tumor conditions while the hafnium synergistically increased radiotherapy efficiency. After particle extravasation via $\mathrm{EPR}$, the particles dissolved in acidic conditions offering an optimal contrast agent for MRI. Besides, the prodrug could be internalized by tumor cells and converted to cisplatin via cellular glutathione. The system provided a dual therapy mechanism (chemo and radiotherapy) effective against a model of breast cancer in vivo (Liu et al., 2017).

Finally, it is worth reporting the work of Li et al. (2020a) that exploited the acidic properties of the tumor microenvironment to increase the generation of ROS in hypoxic tissue, where this strategy is usually not achievable due to the lack of oxygen (Chen et al., 2015). The system was composed of biodegradable magnetic mesoporous nanocubes that efficiently induced hyperthermia when exposed to an external high frequency alternating magnetic field. The particles were loaded with Vitamin $\mathrm{C}$ to selectively kill cancer cells through the formation of the ascorbate radical and $\mathrm{H}_{2} \mathrm{O}_{2}$ (Yun et al., 2015; Lv et al., 2018). Its release was induced by the material phase-change that turned from the solid to the liquid state when the surrounding temperature was higher than $38^{\circ} \mathrm{C}$. More importantly, in acidic conditions, the metallic nature of these nanoparticles induced the transformation of $\mathrm{H}_{2} \mathrm{O}_{2}$ into $\mathrm{O}_{2}$ as well as its further conversion to hydroxyl radical via Fenton reaction (Cao et al., 2018) while serving as an optimal $\mathrm{T}_{2}$ MRI contrast agent. The system showed theranostic properties both in vitro and in vivo, where the particle biodistribution could be tracked via MRI. Upon application of the external magnetic field, the generated hyperthermia induced the release of vitamin $\mathrm{C}$ with its consequent tumor-killing properties.

As shown in this section, $\mathrm{pH}$ responsiveness can be imparted through different chemical linkers and materials sensitive to $\mathrm{pH}$ to trigger the therapeutic and/or the imaging properties. The responsiveness of most of these technologies depends on the sensitivity of their ultrastructure to the $\mathrm{pH}$. More importantly, the $\mathrm{pH}$-responsiveness could be exploited for increasing tumor oxygen levels for improving radiotherapy effectiveness or CDT in hypoxic tumor conditions. Finally, $\mathrm{pH}$-sensitivity can be coupled with other mechanisms of responsiveness that rely on external stimuli to increase the carrier therapeutic and diagnostic properties.

\section{ROS-RESPONSIVE THERANOSTICS}

Due to their high metabolism and accelerated growth, cancer cells are characterized by an increased generation of ROS (Trachootham et al., 2009). On the other hand, this enhanced oxidative stress is compensated by a higher average content of glutathione (Desideri et al., 2019) that in the field of nanotheranostics is exploited as a biological trigger as well, and will be discussed in the last section of this review. It is worth mentioning that all ROS-responsive mechanisms eventually depend on a biological trigger because their working mechanism depends on the oxygen content in the tissue, and they are ineffective in hypoxic regions (Chen et al., 2015). In some cases, the ROS generation is catalyzed directly by the nanoparticles, that due to the properties of their synthesis material, can activate other features of the carriers like payload release (Sun et al., 2018).

In the field of PDT, new evidence are indicating that the best treatment efficacy occurs when the photosensitizer is coupled with a chemotherapeutic agent generating a synergistic effect. 


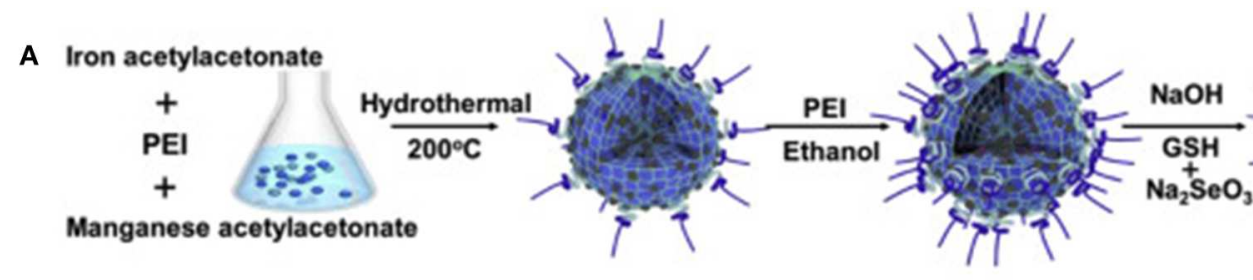

$$
\text { (I) } \mathrm{O}_{2} \stackrel{\mathrm{Se}^{-}}{\longrightarrow} \mathrm{O}_{2}^{-\dagger} \uparrow \stackrel{\mathrm{SOD}}{\longrightarrow} \mathrm{H}_{2} \mathrm{O}_{2} \uparrow \frac{\mathrm{Mn}^{2+}}{\mathrm{ION}} \cdot \mathrm{OH} \dagger
$$
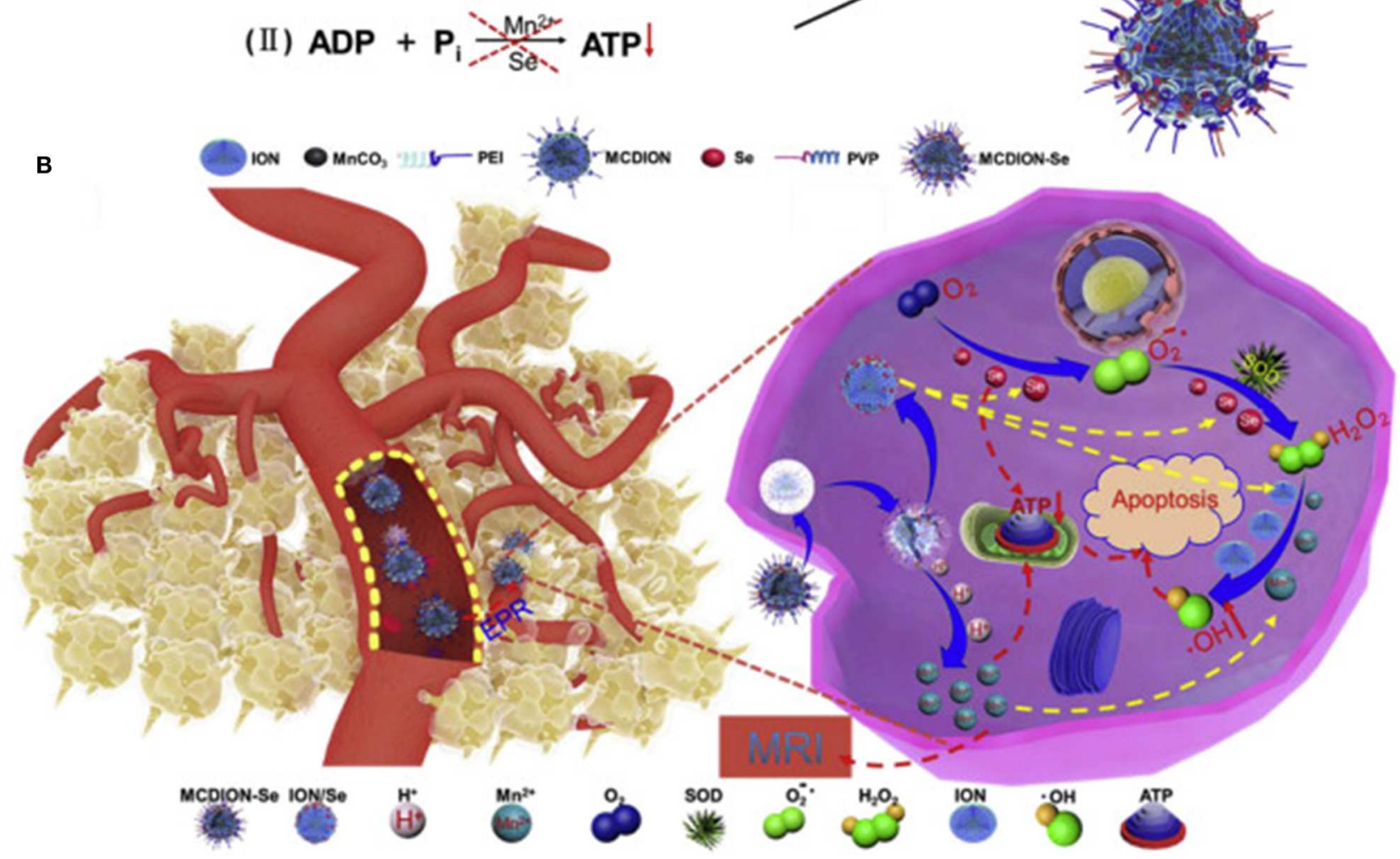

FIGURE 1 | (A) MCDION-Se synthesis. Iron (III) acetylacetonate and manganese acetylacetonate were used as precursors for particle synthesis via solvent, thermal decomposition with polyethyleneimine (PEI) as a surfactant. PEl also allowed for further modification with Se nanoparticles via electrostatic interactions between the positively charged polymer and the negatively charged Se. Polyvinylpyrrolidone (PVP) was used to stabilize the system. The scheme also illustrates the synergistic action of $\mathrm{Mn}$ and $\mathrm{Se}$ in inducing the formation of ROS and consequent cell apoptosis. In particular, the selenium nanoparticles induced the formation of superoxide radicals and the activation of the enzyme superoxide dismutase (SOD) to generate $\mathrm{H}_{2} \mathrm{O}_{2}$. Manganese and iron oxide nanoparticles catalyzed further conversion of $\mathrm{H}_{2} \mathrm{O}_{2}$ to hydroxyl radical. Mn and Se also negatively impacted on ATP synthesis. (B) After IV administration, the particles extravasated in the tumor microenvironment via EPR. Here they can be internalized by cancer cells and induce a cascade of reactions that increase cell apoptosis via ROS production and inhibition of ATP synthesis. Reproduced with permission from Xiao et al. (2019).

To achieve this goal, it is mandatory designing particles in which the ROS can induce a burst release of the therapeutic (Yang et al., 2016a; Zhou et al., 2017). For this reason, the carriers needed to generate ROS as well as being sensitive to these chemical species. To this goal, Sun et al. (2018) developed nanoparticles of pegylated polyphosphate crosslinked with a thioketal linker via $(\mathrm{A} 2+\mathrm{B} 3)$ type polycondensation. The carriers were loaded with the photosensitizer Ce6 and DOX. Under NIR light irradiation, the photosensitizer induced the generation of ROS, while favoring the degradation of the thioketal linker resulting in a burst release of DOX. This strategy was effective in overcoming DOX drug resistance in vitro in a cell line of breast cancer (MCF-7/ADR) overexpressing Pglycoprotein. In vivo, the particle demonstrated good tumor accumulation via the EPR effect as well as high tumor-killing properties. The system showed bimodal imaging capabilities achieved both in vitro and in vivo, exploiting Ce6 photoacoustic (PA) properties and gadolinium (loaded in the particles via its natural affinity for $\mathrm{Ce}$ 6) that allowed for efficient MRI. Supported by dual-modal imaging, the tumor sites could be precisely irradiated, sparing healthy organs and reducing kidney and liver toxicity. 
Within the cell cytoplasm, mitochondria are the most active organelles in terms of ROS generation (Dunn et al., 2015) and, compared to healthy cells, cancer cells showed a higher mitochondrial membrane potential favoring their targeting via internalized cationic molecules (Modica-Napolitano and Aprille, 2001). Inspired by this evidence, Yue et al. developed a nanotheranostic composed of triphenylphosphine, a positively charged molecule with a high affinity for the mitochondria, condensed via amphiphilic block polymerization with camptothecin (CPT) and the photosensitizer zinc phthalocyanine (ZnPC) for PDT generation. The drug conjugation occurred through pegylated thioketal linkers sensitive to ROS (Yue et al., 2016). The particles were designed to extravasate in the tumor microenvironment via EPR, while tumor cell internalization and mitochondrial targeting were achieved through the membrane penetrating properties of the positively charged triphenylphosphine. The ROS generated by the mitochondria and via PDT favored the release of the CPT (topoisomeraseinhibitor) and a dual therapy mode. The system could be detected via fluorescence imaging in vitro and in vivo (when $\mathrm{ZnPC}$ was replaced by $\mathrm{Ce} 6$ ) in a subcutaneous model of lung cancer. However, for a more comprehensive description of remotely responsive activated technologies, we suggest the readers look elsewhere (Kim et al., 2013; Zhang et al., 2016) since this review focuses mostly on the nanoplatforms that are activated by tissueor cell-generated ROS.

In this context, the probe IR790s was recently used to generate a platform named perylene diimide -IR790s-Fe/Pt NPs to detect and trace ROS generation through ratiometric photoacoustic (PA) imaging. Perylene diimide showed a strong NIR light absorption at $680 \mathrm{~nm}$, while IR790 absorbed light at $790 \mathrm{~nm}$. The structure of IR790 could be cleaved by ROS, with a consequent decrease of its adsorption at $790 \mathrm{~nm}$. Ratiometric PA imaging was achieved by irradiating the carriers at 680 and $790 \mathrm{~nm}$. The increase in the $\mathrm{Ab}_{680} / \mathrm{Ab}_{790}$ value was directly proportional to the ROS concentration. ROS generation was facilited by cisplatin conjugated on the surface of the particles via PEG and ferric anions chelated by the perylene diimide. The PEG modification also increased particle biocompatibility and permitted EPR extravasation in vivo. After cell internalization, the cell reductive environment (i.e., glutathione) favored the cisplatin release. Besides its cytostatic effect, it activated the nicotinamide adenine dinucleotide phosphate oxidase (NOX) enzyme that transformed the molecular oxygen in $\mathrm{O}^{-}$furtherly transformed in $\mathrm{H}_{2} \mathrm{O}_{2}$ via superoxide dismutase. The hydrogen peroxide could be further transformed in hydroxyl radical through a reaction catalyzed by the ferric ions inducing effective CDT (Yang et al., 2018b) (Figure 2). The synergistic effect of chemotherapy and CDT was confirmed by an improved in vitro and in vivo tumor cells killing. Qiao et al. (2018) developed a nanotheranostic platform to enhance the temozolomide effect in glioblastoma by reverting the tumor microenvironment immunosuppressive properties through the inhibition of TGF- $\beta$ expression. The carriers were designed to (1) overcome the blood-brain barrier; (2) target to glioma cells; (3) escape from the endolysosomal compartment; (4) co-deliver temozolomide and the siRNA against TGF- $\beta$; (5) be tracked via MRI. The system consisted of superparamagnetic iron nanocubes (an excellent MRI contrast agent) encapsulated in poly[(2-acryloyl)ethyl(p-boronic acid benzyl)diethylammonium bromide] polymer loaded with the TGF- $\beta$ siRNA and coated with zwitterionic lipids coordinating the drug. The particles were additionally modified on their surface with the peptide angiopep-2 targeting low-density lipoprotein receptor-related protein to favor particle translocation through the blood-brain barrier and cancer cell internalization. The system could escape from the endolysosomal compartment through the zwitterionic coating that, in acidic conditions, could acquire a net positive charge destabilizing the membrane of these organelles. Upon interaction with cellular ROS, the benzylboronic acid oxidized reversing the charge of the carriers from positive to negative. This phenomenon induced the release of the therapeutic payloads, and the iron nanoparticles exploited as a contrast agent for MRI.

The application of ROS-responsive theranostics was applied to different conditions. To decrease the generation of $\mathrm{H}_{2} \mathrm{O}_{2}$ in peripheral artery disease that negatively affects the neoangiogenesis process in this condition, Jung et al. generated a new concept of nanotheranostic to detect the diseased tissue through ultrasound and PA imaging (Jung et al., 2019). The system consisted of boronated maltodextrin that, in the presence of tissue $\mathrm{H}_{2} \mathrm{O}_{2}$, released 4-hydroxybenzyl alcohol with proven antioxidant and anti-inflammatory properties (Luo et al., 2017; Tan et al., 2018). Also, upon ROS interaction with the boronate, the system generated $\mathrm{CO}_{2}$ bubbles with echogenic properties for ultrasound imaging. The nanoparticles were loaded with indocyanine green (ICG), allowing for multimodal fluorescent and PA imaging. The theranostic properties of these particles were successfully tested in vitro and in vivo in a model of hindlimb ischemia via intramuscular administration. $\mathrm{H}_{2} \mathrm{O}_{2}$ is considered a mild but very common ROS in hepatic ischemia/reperfusion injury, which is a potentially fatal condition for many conditions, including liver transplantation, liver surgical resection, and hemorrhagic shock (Ushitora et al., 2010). Kang et al. (2016) designed polymeric nanotheranostic based on poly(vanillin oxalate) that can serve as a scavenger for $\mathrm{H}_{2} \mathrm{O}_{2}$ while showing anti-inflammatory and antiapoptotic properties. These particles incorporated a prodrug form of vanillin, a compound known for its anti-inflammatory effect, but not extensively investigated in the clinic due to its short half-life. The molecule was loaded in the particles through $\mathrm{H}_{2} \mathrm{O}_{2}$-sensitive peroxalate ester linkers. More importantly, once activated by $\mathrm{H}_{2} \mathrm{O}_{2}$, the peroxalate esters decomposed in $\mathrm{CO}_{2}$ bubbles that could be tracked via ultrasound imaging in a model of murine hepatic ischemia/reperfusion injury. In this case, the particles were not targeted to exploit their natural tropism toward the liver.

The development of ROS-responsive molecules showed promising results in preclinical testing. Despite their theranostic properties, they can also serve as in vivo nanosensors to measure ROS generation. The responsiveness of some material to ROS can be exploited to generate $\mathrm{CO}_{2}$ for improving current ultrasound diagnostic methods. Besides, these technologies can be applied to different diseases, since the generation of ROS is a characteristic of various pathological conditions. 


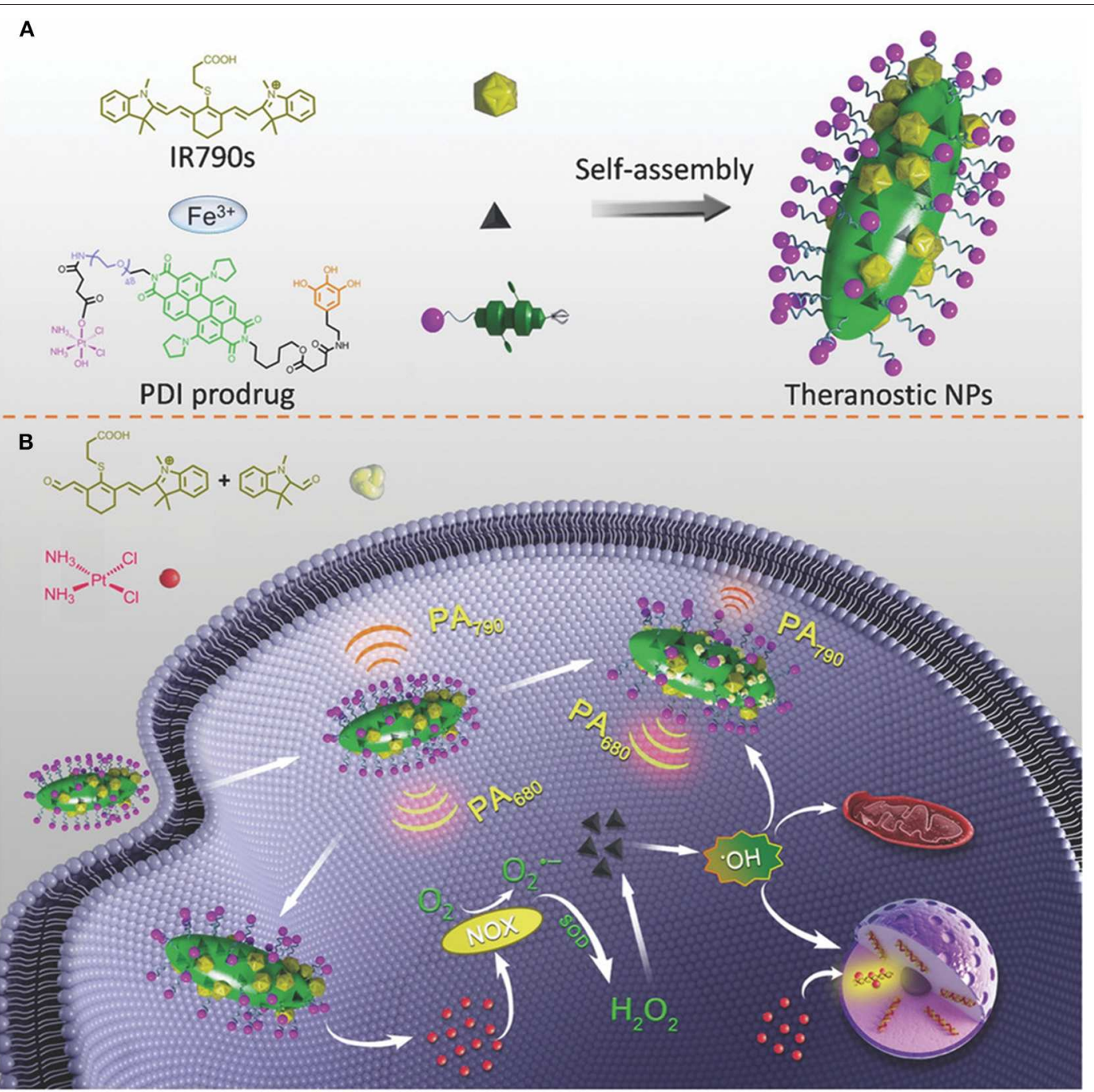

FIGURE 2 | (A) Self-assembly of perylene diimide cisplatin prodrug and the infrared dye IR790 in the presence of ferric ions. One of the amide groups of the PDI was modified with polyphenols coordinating the ferric ions necessary for catalyzing $\mathrm{H}_{2} \mathrm{O}_{2}$ in hydroxyl radical in acidic conditions. The second amide of the PDI was conjugated with PEG that increased nanoparticle solubility and allowed for further modification with the cisplatin prodrug. (B) The working mechanism of the system. After cancer cell internalization, the cisplatin induced the activation of the nicotinamide adenine dinucleotide phosphate oxidase (NOX) transforming molecular oxygen in $\mathrm{O}^{-}$with consequent generation of $\mathrm{H}_{2} \mathrm{O}_{2}$ via superoxide dismutase. The hydrogen peroxide is further transformed into hydroxyl radicals by the ferric ions inducing cell apoptosis. ROS formation degraded IR790. The measurement of the perylene diimide/IR790 absorption ratio could be used for ratiometric PA imaging of the ROS formation. Reproduced with permission from Yang et al. (2018b).

\section{ENZYME RESPONSIVE THERANOSTICS}

Tissue remodeling and the overexpression of the lytic enzymes that govern this process characterize many pathological conditions. These enzymes can be exploited as triggers since they can favor the degradation of the carriers and the consequent release of the theranostic payloads in the diseased area.

In the case of enzyme-responsive theranostics, are not rare examples of nanoparticles with multiple responsive properties. For instance, Chen et al. developed ferritin nanocages sensitive to $\mathrm{pH}$ and matrix metalloproteinase (MMP)-13 activity. The particles, named CMFn@HCQ, were conceived to deliver hydroxychloroquine in the cartilage tissue to ameliorate osteoarthritis conditions (Chen et al., 2019b). In osteoarthritis, the joint microenvironment is characterized by an acidic $\mathrm{pH}$ (close to 6) (Li et al., 2017) and MMP-13 overexpression. Ferritin was genetically modified to increase its targeting for collagen II through the addition of a specific peptide in its structure. These carriers were further modified with an MMP-13 cleavable peptide conjugated with the near-infrared (NIR) dye cy5.5 and a quencher to provide the system with enzyme-sensitive diagnostic properties. Because of this chemical modification, only after MMP-13 activity separating the dye and the quencher, the diagnostic signal could be registered. The particles were loaded with the anti-inflammatory hydroxychloroquine, and their structure degraded under acidic conditions inducing the 
release of the payload. The resulting nanocages had a size of $20 \mathrm{~nm}$, favoring their diffusion in the dense protein matrix of the joint. These carriers demonstrated high therapeutic properties as well as the ability to detect MMP-13 overexpression in vitro and in vivo when administered via intra-articular injections. Ren et al. (2019) engineered a carrier named HA-Ce6 DOX composed of a hyaluronic acid ultrastructure that, despite the ability to offer a natural targeting for the CD44 receptor, overexpressed on cancer cells, also granted the presence of multiple modification sites. The system was conjugated with Ce6 (for PDT and fluorescent detection) and DOX through a $\mathrm{pH}$-sensitive hydrazine bond (Figure 3). The release of the photosensitizer and the chemotherapeutic was further accelerated by the enzyme hyaluronidase, overexpressed in the tumor microenvironment. The enzymatic degradation of the particles was fundamental for the activation of the diagnostic properties of the system since, when encapsulated, Ce6 was affected by the aggregation-caused quenching (ACQ) effect (Li et al., 2020b). CPT-loaded mesoporous silica nanoparticles were functionalized with a cyclo-RGD peptide, and another peptide conjugated with a fluorescent dye and a quencher. Both the peptides were sensitive to the proteolytic action of MMP2. Cyclo-RGD had the function of improving cancer cell targeting and stabilizing the drug in the carriers' pores. When the particles were internalized in the cells, both the peptides were digested inducing the quencher and the dye separation with consequent fluorescent signal detection and drug release (Hu et al., 2016). More investigation will be necessary to test the efficacy of this theranostic platform in vivo. Gold nanorods were used to generate a dual stimuli theranostic nanocarrier to provide efficient PTT as well as a detectable diagnostic signal in response to tumor $\mathrm{pH}$ and MMPs (Zhao et al., 2017). The gold nanorods were modified with an asymmetric cyanine via an MMP sensible linker. This dye could emit near-infrared fluorescence in a pH-dependent manner (Zhao et al., 2015), presenting a reversible "off/on" signal emission as a function of this parameter. Despite its sensitivity for the $\mathrm{pH}$, the gold nanorods represented a significant FÖrster resonance energy transfer (FRET) quencher for the asymmetric cyanine that could emit the fluorescent signal only when in free form. Both the gold nanorods and the asymmetric cyanine allowed for PTT upon irradiation at $808 \mathrm{~nm}$. Finally, drug delivery was achieved through an additional functionalization with glycosyl groups that significantly increased particle biocompatibility, EPR effect, and tumor targeting via GLUT-1 receptor. Liu et al. (2016b) designed a nanotheranostic system based on fluorescent quantum dots embedded in a nanoporous silica matrix with $\mathrm{pH}$-activatable targeting properties and a protease-sensitive drug delivery mechanism. The system was surface modified with a zwitterionic anti-biofouling layer composed of chemical groups with positive and negative charges $\left[\mathrm{COO}^{-}\right.$and $-\mathrm{HN}{ }^{+}$ (Me), respectively]. In physiological $\mathrm{pH}$ conditions, the particles showed high circulation time, reduced sequestration in the organ of the mononuclear phagocytic system, and reduced protein corona formation due to their neutral surface charge. To this purpose, this strategy was previously demonstrated to be more effective and stable than PEG surface modification (Holmlin et al., 2001; Gui et al., 2013). When exposed to $\mathrm{pH}$ below 6.8, like in the tumor microenvironment, they acquired a positive charge favoring their uptake into the tumor cells. The pores of the silica could be loaded with a drug (DOX) and coated with the polymer polycaprolactone that was exploited to stabilize the drug in the particle structure. This polymer was sensitive to the enzyme esterase that is overexpressed and secreted by cancer cells, making tumor microenvironment and cells favorable sites for drug release. Also, it is important to highlight that the enzymatic degradation was further favored at acidic $\mathrm{pH}$, where the particles acquired a positive charge and were more accessible to the enzyme and prone to cell internalization.

The enzyme-responsive theranostic working mechanism usually depends on hydrolytic enzymes favoring carrier or peptide linkers degradation. This phenomenon can also be exploited through coatings used to stabilized the therapeutics or the diagnostic molecules in the particles. The efficient application of enzyme-responsive theranostics strictly depends on the over-expression of some enzymes that can characterize different pathological conditions. Their synthesis relies on the engineering of biological substrates, usually functionalized with other molecules (including targeting modifications). Some of these modifications aim at providing higher biocompatibility and biological interaction, opening new avenues of research in the field of bioinspired nanomedicine.

\section{THERANOSTICS RESPONSIVE TO OTHER BIOLOGICAL STIMULI}

Glutathione (GSH) is a reactive molecule controlling the cellular redox balance. Cancer cells significantly overexpress this molecule, pointing out its potential role as a targetable trigger for the development of smart nanotheranostics (Liu et al., 2016a). To this purpose, theranostic platforms based on GSH reactivity are usually composed of a targeted particle modified with a disulfide bond coordinating a chemotherapeutic and/or a fluorescent probe (Han et al., 2017). A typical example of GSH-responsive theranostic based on a facile synthesis is represented by nanoparticles generated through hydrophilic polymers conjugated with hydrophobic drugs. The amphiphilic monomers can favor nanoparticle self-assembly, leaving room for further modification with a diagnostic probe. Exploiting these principals, Li et al. (2019b) generated multimodal imaging and therapeutic nanotheranostics named DHP. These carriers consisted of a disulfide-bond-linked hydroxyethyl starch conjugated with the chemotherapeutic paclitaxel (PXT). The addition of dioctadecyl-3,3,3,3-tetramethylindotricarbocyanine iodide (DiR) in the synthesis solution resulted in the entrapment of the dye in the hydrophobic core of the particles through one single step of dialysis. DiR allowed for both fluorescent and PA imaging, and when irradiated with at $808 \mathrm{~nm}$, PTT providing synergistic effects with the chemotherapeutic. When packaged within the nanoparticle structure, the dye was affected by ACQ. However, after particle internalization, the cellular GSH induced the release of the theranostic payloads (Li et al., 2019b) (Figure 4). These properties were proven in vitro and in vivo 

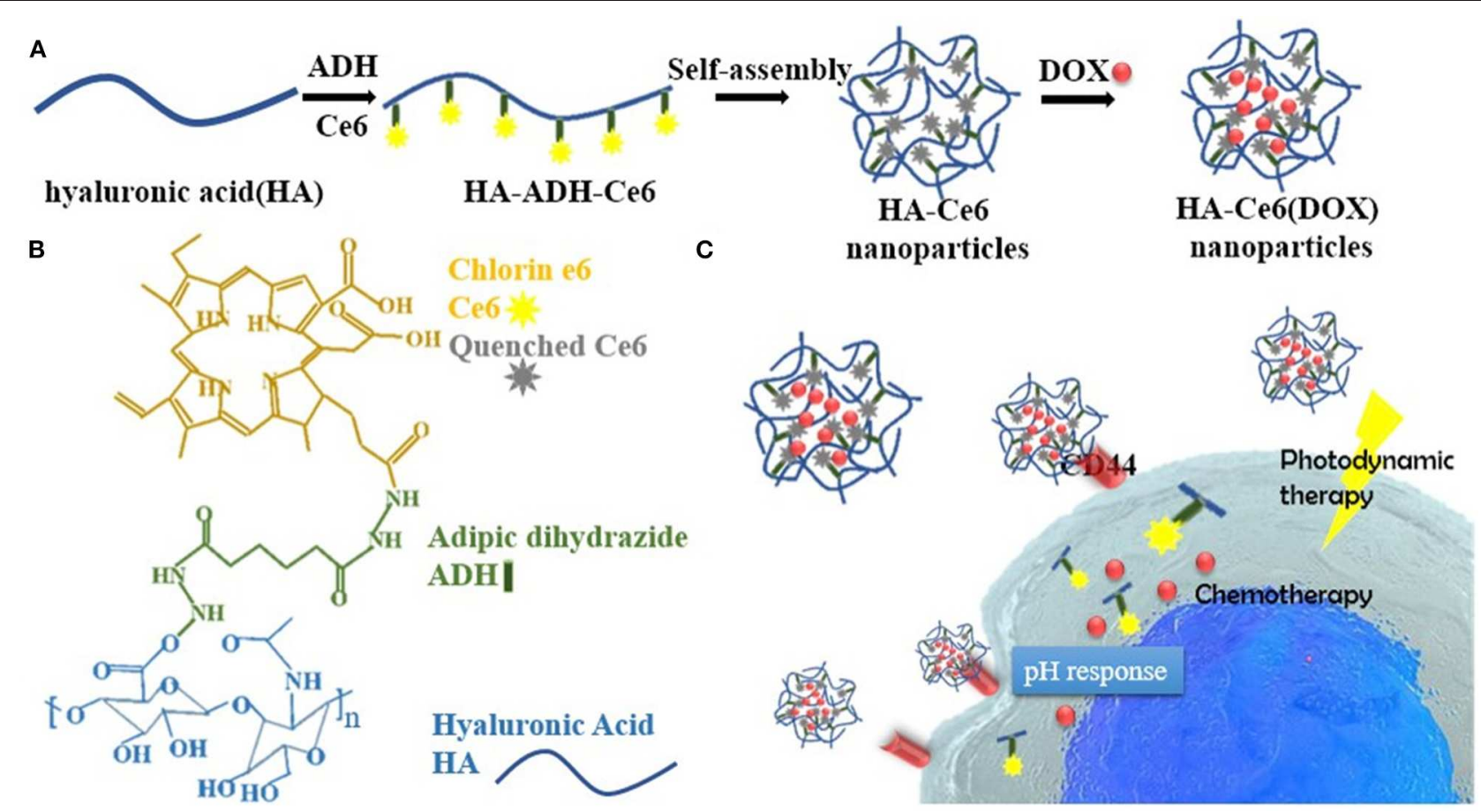

FIGURE 3 | (A) Synthetic process of HA-Ce6 (DOX) nanoparticles. Hyaluronic acid (HA) and adipic dihydrazide (ADH) were dissolved in the presence of Ce6-NHS ester and EDCl catalyzing the reaction of particle self-assembly in acidic conditions. Doxorubicin (DOX) loading was performed post-synthesis. (B) Illustration of the different components forming the particles. (C) the working mechanism of HA-Ce6 (DOX). After intravenous injection, the particles targeted the tumor via EPR and were internalized by the cancer cells through the CD44 HA receptor. In tumor microenvironment and cells, the particle could dissolve releasing DOX and Ce6, whose fluorescence was previously inhibited by the presence of a quencher in particle structure. Particle degradation was favored by the acidic conditions acting on the $\mathrm{pH}$-sensitive hydrazine linkers and by the enzyme hyaluronidase degrading the nanoparticle matrix. Reproduced with permission from Ren et al. (2019).

in a model of breast cancer. These particles could efficiently accumulate in the tumor via EPR due to the high circulation properties offered by the hydroxyethyl starch. Similarly, a GSHsensitive platform was synthesized through low molecular weight heparin polymerization that, in the presence of cystamine, polymerized with $\mathrm{Ce} 6$ in biocompatible carriers (Yang et al., 2018a). In addition, heparin was previously shown to possess antimetastatic and anti-angiogenic properties (Yang et al., 2015; Mei et al., 2017). The system offered sensitivity to a reductive environment since the cystamine linker contained a disulfide bond in its structure. GSH induced particle degradation and Ce6 release activating the theranostic properties of the dye that, when encapsulated, was affected by the ACQ effect. Finally, the system was also loaded with PXT in combination with alpha-tocopherol succinate (Yang et al., 2018a) to provide therapeutic synergism between chemotherapy and PDT. When injected intravenously, the particles accumulated via EPR in a model of $4 \mathrm{~T} 1$ breast cancer. After cell internalization, the particles were degraded by the abundant levels of GSH releasing PXT and Ce6 that in free form was easily detectable through NIR imaging and inducible for PDT. Song et al. (2017) engineered iron oxide nanoparticles to be responsive to ATP, which is highly generated in tumor cells when compared to healthy tissue as well as to a decrease in tissue $\mathrm{pH}$. The system was composed of small clusters of superparamagnetic iron nanoparticles and tannic acid that self-assembled in water-soluble aggregates smaller than $100 \mathrm{~nm}$ due to hydrophobic interactions. The particles were modified with ICG and DSPE-PEG via non-covalent hydrophobic interactions and hydrogen bonds, respectively. The presence of PEG increased particle circulation and tumor accumulation via EPR. The core of iron oxide permitted strong MRI properties while its high affinity for ATP induced particle disassembly in the presence of this molecule (Yu et al., 2013). On the other hand, the ICG permitted particle fluorescence detection after degradation (since the dye was affected by the ACQ effect) as well as PTT applications when irradiated at $808 \mathrm{~nm}$. Particle degradation was further boosted in acidic pH, perhaps due to the protonation of the hydroxyl groups of the tannic acid that lowered the affinity of this molecule for the iron oxide nanoparticle structure. The disassembly of the system was pivotal to activate its imaging properties as well as its renal clearance. The system was successfully tested in vitro and in vivo in a subcutaneous tumor model for its theranostic and responsiveness properties obtained through facile synthesis and loading protocols.

Biological responsiveness was also used to ameliorate the conditions of diseases different than cancer. In Alzheimer's disease, the aberrant generation of plaques of soluble $\beta$-Amyloid proteins in the central nervous system occurs (Benilova et al., 2012). This phenomenon is favored by the accumulation of 


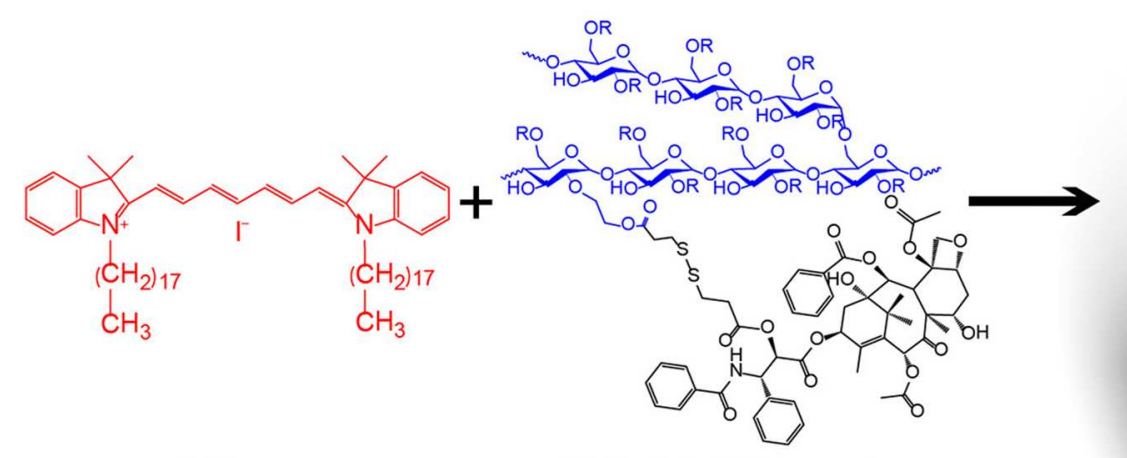

DiR

HES-SS-PTX conjugate

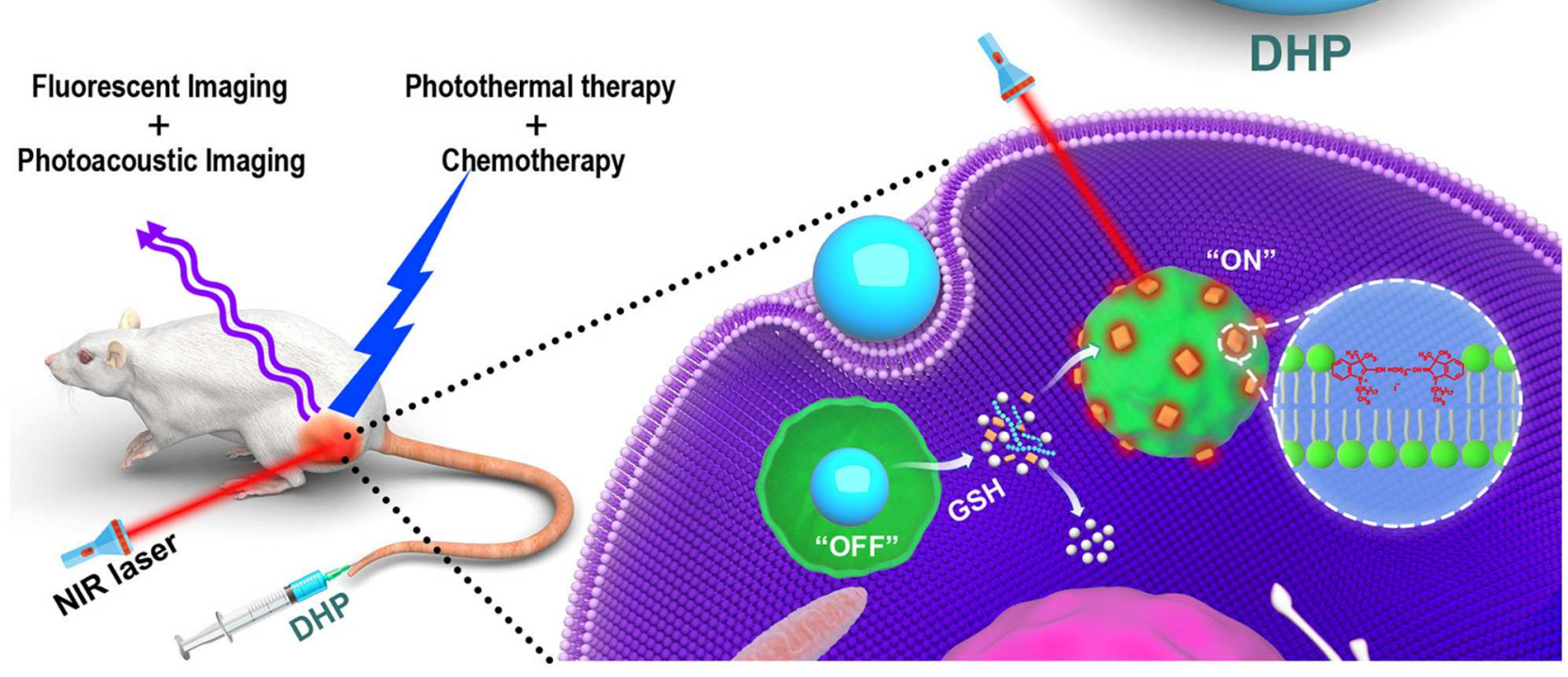

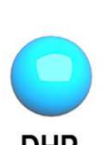

DHP

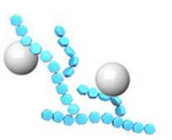

HES-SS-PTX conjugate

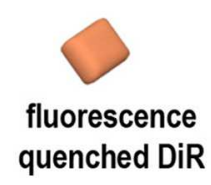

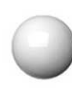

PTX
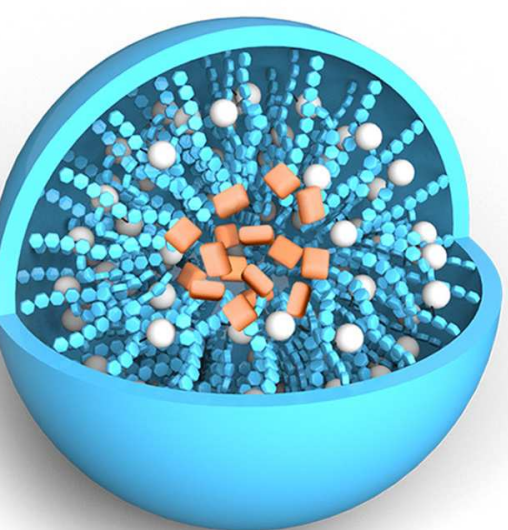

DHP

FIGURE 4 | The major components of DHP nanoparticles are the disulfide-bond-linked hydroxyethyl starch paclitaxel conjugate (HES-SS-PTX) and the near-infrared dye cyanine fluorophore DiR. The particles were designed to exert dual imaging (fluorescent and photoacoustic imaging) and dual therapy (photothermal and chemotherapy) properties. The HES shell provided the particles with EPR properties. After intravenous injection, the particles could accumulate in the tumor microenvironment and be internalized inside the cells. In the cell cytoplasm, the disulfide bond degradation occurred due to the cellular GSH that favored the release of PXT (chemotherapy) and DiR. When encapsulated in the particles, DiR was affected by ACQ, but upon its release, it could exert its therapeutic (photothermal therapy) and diagnostic (photoacoustic and fluorescent imaging) properties. Reproduced with permission from Li et al. (2019b).

metal ions in the tissue like copper, which via ROS generation can support protein aggregation (Atwood et al., 2018). Cui et al. designed upconversion NaYF4:Yb/Er/Tm nanocrystals conjugated with 8-hydroxyquinoline-2-carboxylic (HQC) and DSPE-PEG to provide the carriers with diagnostic, therapeutic and biocompatibility properties, respectively (Cui et al., 2016). The particles were designed to (1) detect $\mathrm{Cu}^{2+}$ via luminescence resonance energy transfer from the particle to the copper, (2) targeting and imaging of the $\beta$-Amyloid- $\mathrm{Cu}^{2+}$ complexes by registering the upconverted light signal emitted further NIR light irradiation (demonstrated in vitro and ex-vivo) (3) chelate the copper via HCQ. The theranostic properties of this system were successfully proven in a zebrafish model and ex-vivo.

These examples demonstrated that any reactive molecules could be exploited as a tool to generate responsive nanoparticles. GSH, in particular, was extensively investigated in literature for this purpose since cancer cells are characterized by a very high content of this molecule. On the other hand, they are also characterized by the high generation of ROS. For this reason, further advances in the field need to take into consideration the 
TABLE 1 | Summary table of the different technologies and their theranostic properties.

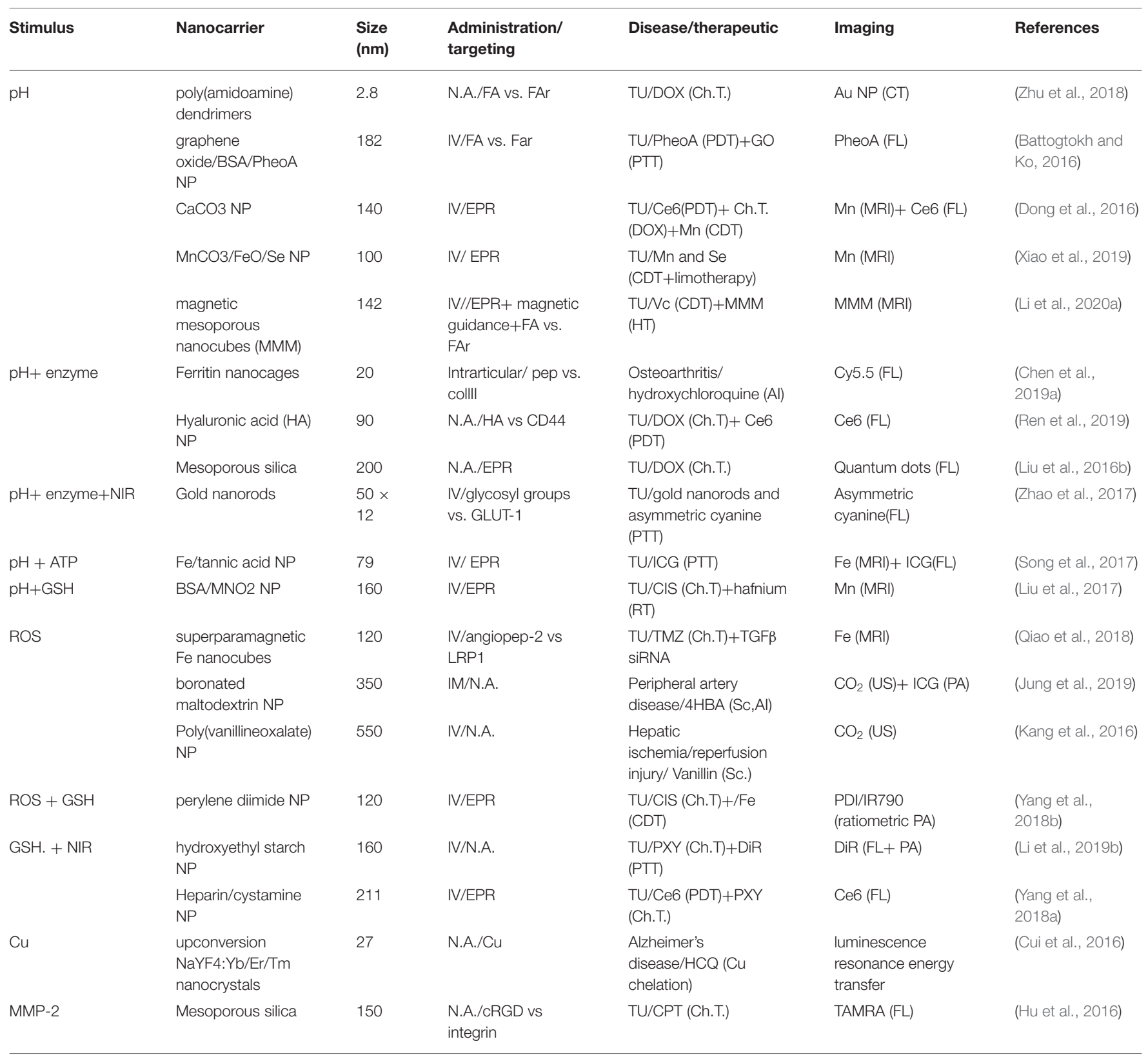

Al, anti inflammatory; Ch.T., Chemotherapy; CDT, Chemodynamic therapy; CPT, Camptothecin; DOX, Doxorubicin; EPR, Enhanced permeability and retention effect; FA, Folic acid; Far, Folic acid receptor; FL, Fluorescence; GO, Graphen Oxide; GSH, Glutathione; HA, Hyaluronic acid; HT, Hyperthermal therapy; IV, intravenous; LRP1, Iow-density lipoprotein receptor

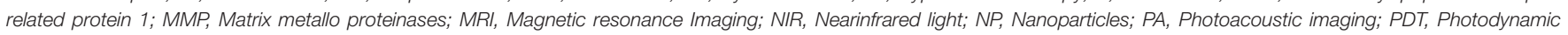
tehrapy; Pep, peptide; PTT, Photheral therapy; PXT, Paclitaxel; ROS, Reactive oxygen species; RT, Radiotherapy; Sc, scavenger; TU, Tumor; US, Ultrasound imaging.

efficient alteration of this balance, both when generating GSH- as well as ROS-responsive carriers.

\section{CONCLUSIONS}

The fine-tuning of the therapeutic regimens and diagnostic methods represents an emerging need in all the fields of experimental medicine. Current efforts of the scientific community are paving the way to achieve this goal through the generation of materials that are sensitive and can respond to chemical, physical, and biological triggers. Therapeutic and diagnostic properties can reside in the same molecule embedded in the nanocarrier structure that can be activated at the injury site, or they can independently derive from different chemicals loaded into and/or conjugated onto the surface of the nanoparticles. Also, the very same structure of the particles can alternatively provide therapeutic or diagnostic properties. In this work, we presented some examples of pathological stimuli-responsive theranostics with multiple therapeutic and diagnostic properties, 
sometimes combined with external stimuli-responsive materials (Table 1). The goal of this work was to present the current strategies and materials used for imparting the theranostics with biological-responsiveness.

To our knowledge, no nanotheranostic was FDA approved to date (Anselmo and Mitragotri, 2019). However, clinical trials are currently active to evaluate their efficacy and safety. In this effort, most of the theranostic under clinical trials base their working mechanisms on remotely applied stimuli. In particular, iron (Nardecchia et al., 2019) and gold (Pedrosa et al., 2015) nanoparticles are extensively tested in humans for their multimodal imaging modalities, PDT and PTT properties as well as for their relatively easy synthesis and functionalization. External stimuli-responsive carriers activation strongly depends on the penetration and the precision of the external signal, and most importantly, they can be activated only in the areas that current diagnostic tools detect as disease sites. On the other hand, the responsiveness of theranostics to pathological stimuli theoretically depends solely on their ability to target the environmental conditions of the diseased tissue or cell, with no further interventions of external procedures. In this context, they could reveal and be activated in sick areas that were not detected by other imaging tools. Precise targeting, therefore, represents their main limitation. Due to the complex nature of these technologies, targeting is usually imparted through the pegylation of the carriers and depends on their passive accumulation in the diseased tissue through the EPR effect. However, this kind of targeting could

\section{REFERENCES}

Anselmo, A. C., and Mitragotri, S. (2019). Nanoparticles in the clinic: an update. Bioeng. Trans. Med. 4:e10143. doi: 10.1002/btm2.10143

Atwood, C. S., Huang, X., Moir, R. D., Tanzi, R. E., and Bush, A. I. (2018). "Role of free radicals and metal ions in the pathogenesis of alzheimer's disease," in Metal Ions in Biological Systems (Routledge: CRC Press, Taylor and Francis publishing group- New york), 309-364. doi: 10.1201/9780203747605-10

Battogtokh, G., and Ko, Y. T. (2016). Graphene oxide-incorporated pHresponsive folate-albumin-photosensitizer nanocomplex as image-guided dual therapeutics. J. Control. Release 234, 10-20. doi: 10.1016/j.jconrel.2016.05.007

Benilova, I., Karran, E., and De Strooper, B. (2012). The toxic A $\beta$ oligomer and alzheimer's disease: an emperor in need of clothes. Nat. Neurosci. 15, 349-57. doi: $10.1038 / \mathrm{nn} .3028$

Cao, Z., Li, W., Liu, R., Li, X., Li, H., Liu, L., et al. (2019). pH-and enzyme-triggered drug release as an important process in the design of anti-tumor drug delivery systems. Biomed. Pharmacother. 118:109340. doi: 10.1016/j.biopha.2019.109340

Cao, Z., Zhang, L., Liang, K., Cheong, S., Boyer, C., Gooding, J. J., et al. (2018). Biodegradable 2D Fe-Al hydroxide for nanocatalytic tumor-dynamic therapy with tumor specificity. Adv. Sci. 5:1801155. doi: 10.1002/advs.201801155

Chen, C., Gao, K., Lian, H., Chen, C., and Yan, X. (2019a). Singleparticle characterization of theranostic liposomes with stimulus sensing and controlled drug release properties. Biosens. Bioelectron. 131, 185-192. doi: 10.1016/j.bios.2019.02.016

Chen, H., Qin, Z., Zhao, J., He, Y., Ren, E., Zhu, Y., et al. (2019b). Cartilage-targeting and dual MMP-13/pH responsive theranostic nanoprobes for osteoarthritis imaging and precision therapy. Biomaterials 225:119520. doi: 10.1016/j.biomaterials.2019.1 19520 jeopardize their responsiveness. For example, in some cases, the carriers need to be internalized in the cells to perform their theranostic functions while PEG functionalization could affect this process (Moros et al., 2012). The complexity of their synthesis protocols, as well as their low biocompatibility and inherent toxicity, represent other significant limitations in the clinical translation of these technologies (Kunjachan et al., 2015). However, more investigations on the development of theranostic molecules like porphyrins, $\mathrm{Ce} 6$, or $\mathrm{Mn}^{2+}$ could streamline their synthetic routes while combining the advantages of external- and biological-responsive theranostics. Finally, relying on biological stimuli, they could suffer from unspecific activation in other tissues (i.e., the organs of the mononuclear phagocytic system). For this reason, more research to evaluate their adverse effect, metabolization, and excretion is necessary for future developments.

\section{AUTHOR CONTRIBUTIONS}

AP and YA: conceptualization. AP and MR writing (original draft preparation). SL review and editing. AZ supervision and funding acquisition.

\section{FUNDING}

This research was supported by the Russian Science Foundation (grant \# 16-15-10410) and by the Russian Academic Excellence Project 5-100.

Chen, H., Tian, J., He, W., and Guo, Z. (2015). $\mathrm{H}_{2} \mathrm{O}_{2}$-activatable and $\mathrm{O}_{2}$-evolving nanoparticles for highly efficient and selective photodynamic therapy against hypoxic tumor cells. J. Am. Chem. Soc. 137, 1539-1547. doi: 10.1021/ja511420n

Cui, Z., Bu, W., Fan, W., Zhang, J., Ni, D., Liu, Y., et al. (2016). Sensitive imaging and effective capture of $\mathrm{Cu} 2+$ : towards highly efficient theranostics of alzheimer's disease. Biomaterials 104, 158-167. doi: 10.1016/j.biomaterials.2016.06.056

Das, T. K., Wati, M. R., and Fatima-Shad, K. (2015). Oxidative stress gated by fenton and haber weiss reactions and its association with alzheimer's disease. Arch. Neurosci. 2:e60038. doi: 10.5812/archneurosci.20078

Desideri, E., Ciccarone, F., and Ciriolo, M. R. (2019). Targeting glutathione metabolism: partner in crime in anticancer therapy. Nutrients 11:1926. doi: 10.3390/nu11081926

Dong, Z., Feng, L., Zhu, W., Sun, X., Gao, M., Zhao, H., et al. (2016). $\mathrm{CaCO}_{3}$ nanoparticles as an ultra-sensitive tumor-pH-responsive nanoplatform enabling real-time drug release monitoring and cancer combination therapy. Biomaterials 110, 60-70. doi: 10.1016/j.biomaterials.2016.09.025

Dunn, J. D., Alvarez, L. A., Zhang, X., and Soldati, T. (2015). Reactive oxygen species and mitochondria: a nexus of cellular homeostasis. Redox Biol. 6, 472-485. doi: 10.1016/j.redox.2015.09.005

Fan, Z., Sun, L., Huang, Y., Wang, Y., and Zhang, M. (2016). Bioinspired fluorescent dipeptide nanoparticles for targeted cancer cell imaging and real-time monitoring of drug release. Nat. Nanotechnol. 11, 388-94. doi: 10.1038/nnano.2015.312

Gui, A. L., Luais, E., Peterson, J. R., and Gooding, J. J. (2013). Zwitterionic phenyl layers: finally, stable, anti-biofouling coatings that do not passivate electrodes. ACS Appl. Mater. Interfaces 5, 4827-4835. doi: 10.1021/am400519m

Han, L., Zhang, X.-Y., Wang, Y.-L., Li, X., Yang, X.-H., Huang, M., et al. (2017). Redox-responsive theranostic nanoplatforms based on inorganic nanomaterials. J. Control. Release 259, 40-52. doi: 10.1016/j.jconrel.2017.03.018 
Holmlin, R. E., Chen, X., Chapman, R. G., Takayama, S., and Whitesides, G. M. (2001). Zwitterionic SAMs that resist nonspecific adsorption of protein from aqueous buffer. Langmuir 17, 2841-2850. doi: 10.1021/la0015258

Hu, J.-J., Liu, L.-H., Li, Z.-Y., Zhuo, R.-X., and Zhang, X.-Z. (2016). MMPresponsive theranostic nanoplatform based on mesoporous silica nanoparticles for tumor imaging and targeted drug delivery. J. Mater. Chem. B 4, 1932-1940. doi: 10.1039/C5TB02490K

Idris, S., Arifah, A., Jesse, F., Ramanoon, S., Basit, M., Zakaria, Z., et al. (2019). Synthesis, characterization, and in vitro release of oxytetracycline loaded in pH-responsive $\mathrm{CaCO}_{3}$ nanoparticles. J. Appl. Pharm. Sci. 9, 019-027. doi: 10.7324/JAPS.2019.91103

Jo, S. D., Ku, S. H., Won, Y.-Y., Kim, S. H., and Kwon, I. C. (2016). Targeted nanotheranostics for future personalized medicine: recent progress in cancer therapy. Theranostics 6, 1362-1377. doi: 10.7150/thno.15335

Jung, E., Lee, J., Jeong, L., Park, S., Lee, M., Song, C., et al. (2019). Stimulusactivatable echogenic maltodextrin nanoparticles as nanotheranostic agents for peripheral arterial disease. Biomaterials 192, 282-291. doi: 10.1016/j.biomaterials.2018.11.022

Kanamala, M., Wilson, W. R., Yang, M., Palmer, B. D., and Wu, Z. (2016). Mechanisms and biomaterials in $\mathrm{pH}$-responsive tumour targeted drug delivery: a review. Biomaterials 85, 152-167. doi: 10.1016/j.biomaterials.2016.01.061

Kang, C., Cho, W., Park, M., Kim, J., Park, S., Shin, D., et al. (2016). $\mathrm{H}_{2} \mathrm{O}_{2}$-triggered bubble generating antioxidant polymeric nanoparticles as ischemia/reperfusion targeted nanotheranostics. Biomaterials 85, 195-203. doi: 10.1016/j.biomaterials.2016.01.070

Karimi, M., Ghasemi, A., Zangabad, P. S., Rahighi, R., Basri, S. M. M., Mirshekari, H., et al. (2016). Smart micro/nanoparticles in stimulus-responsive drug/gene delivery systems. Chem. Soc. Rev. 45, 1457-1501. doi: 10.1039/C5CS00798D

Kim, T. H., Lee, S., and Chen, X. (2013). Nanotheranostics for personalized medicine. Expert Rev. Mol. Diagn. 13, 257-269. doi: 10.1586/erm.13.15

Kunjachan, S., Ehling, J., Storm, G., Kiessling, F., and Lammers, T. (2015). Noninvasive imaging of nanomedicines and nanotheranostics: principles, progress, and prospects. Chem. Rev. 115, 10907-10937. doi: 10.1021/cr500314d

Li, C., Li, H., Wang, Q., Zhou, M., Li, M., Gong, T., et al. (2017). pH-sensitive polymeric micelles for targeted delivery to inflamed joints. J. Control. Release 246, 133-141. doi: 10.1016/j.jconrel.2016.12.027

Li, F., Liang, Z., and Ling, D. (2019a). Smart organic-inorganic nanogels for activatable theranostics. Curr. Med. Chem. 26, 1366-1376. doi: 10.2174/0929867324666170920164614

Li, J., Liu, Y., Li, X., Liang, G., Ruan, C., and Cai, K. (2020a). ROS self-generation and hypoxia self-enhanced biodegradable magnetic nanotheranostics for targeted tumor therapy. Nanoscale Horizons. 5, 350-358 doi: 10.1039/C9NH00490D

Li, J., Wang, J., Li, H., Song, N., Wang, D., and Tang, B. Z. (2020b). Supramolecular materials based on AIE luminogens (AIEgens): construction and applications. Chem. Soc. Rev. 49, 1144-1172. doi: 10.1039/C9CS00495E

Li, Y., Wu, Y., Chen, J., Wan, J., Xiao, C., Guan, J., et al. (2019b). A simple glutathione-responsive turn-on theranostic nanoparticle for dualmodal imaging and chemo-photothermal combination therapy. Nano Lett. 19, 5806-5817. doi: 10.1021/acs.nanolett.9b02769

Lin, H., Chen, Y., and Shi, J. (2018). Nanoparticle-triggered in situ catalytic chemical reactions for tumour-specific therapy. Chem. Soc. Rev. 47, 1938-1958. doi: 10.1039/C7CS00471K

Liu, D., Yang, F., Xiong, F., and Gu, N. (2016a). The smart drug delivery system and its clinical potential. Theranostics 6, 1306-1323. doi: 10.7150/thno.14858

Liu, J., Chen, Q., Zhu, W., Yi, X., Yang, Y., Dong, Z., et al. (2017). Nanoscalecoordination-polymer-shelled manganese dioxide composite nanoparticles: a multistage Redox $/ \mathrm{pH} / \mathrm{H}_{2} \mathrm{O}_{2}$-responsive cancer theranostic nanoplatform. $A d v$. Func. Mater. 27:1605926. doi: 10.1002/adfm.201605926

Liu, J., Huang, Y., Kumar, A., Tan, A., Jin, S., Mozhi, A., et al. (2014a). pH-sensitive nano-systems for drug delivery in cancer therapy. Biotechnol. Adv. 32, 693-710. doi: 10.1016/j.biotechadv.2013.11.009

Liu, P., Yue, C., Sheng, Z., Gao, G., Li, M., Yi, H., et al. (2014b). Photosensitizerconjugated redox-responsive dextran theranostic nanoparticles for nearinfrared cancer imaging and photodynamic therapy. Polym. Chem. 5, 874-881. doi: 10.1039/C3PY01173A

Liu, Z., Chen, X., Zhang, X., Gooding, J. J., and Zhou, Y. (2016b). Carbon-quantum-dots-loaded mesoporous silica nanocarriers with $\mathrm{pH}$ switchable zwitterionic surface and enzyme-responsive pore-cap for targeted imaging and drug delivery to tumor. Adv. Healthc. Mater. 5, 1401-1407. doi: 10.1002/adhm.201600002

Luo, L., Kim, S.-W., Lee, H.-K., Kim, I.-D., Lee, H., and Lee, J.-K. (2017). Anti-oxidative effects of 4-hydroxybenzyl alcohol in astrocytes confer protective effects in autocrine and paracrine manners. PLoS ONE 12:e177322. doi: 10.1371/journal.pone.0177322

Lv, H., Wang, C., Fang, T., Li, T., Lv, G., Han, Q., et al. (2018). Vitamin C preferentially kills cancer stem cells in hepatocellular carcinoma via SVCT-2. NPJ Precis. Oncol. 2:1. doi: 10.1038/s41698-017-0044-8

Ma, Y., Huang, J., Song, S., Chen, H., and Zhang, Z. (2016). Cancer-targeted nanotheranostics: recent advances and perspectives. Small 12, 4936-4954. doi: 10.1002/smll.201600635

Mei, L., Liu, Y., Xia, C., Zhou, Y., Zhang, Z., and He, Q. (2017). Polymerdrug nanoparticles combine doxorubicin carrier and heparin bioactivity functionalities for primary and metastatic cancer treatment. Mol. Pharm. 14, 513-522. doi: 10.1021/acs.molpharmaceut.6b00979

Modica-Napolitano, J. S., and Aprille, J. R. (2001). Delocalized lipophilic cations selectively target the mitochondria of carcinoma cells. Adv. Drug Deliv. Rev. 49, 63-70. doi: 10.1016/S0169-409X(01)00125-9

Moros, M., HernáEz, B., Garet, E., Dias, J. T., SáEz, B., Graz,ú, V., et al. (2012). Monosaccharides versus PEG-functionalized NPs: influence in the cellular uptake. ACS Nano 6, 1565-1577. doi: 10.1021/nn20 $4543 \mathrm{c}$

Nardecchia, S., Sánchez-Moreno, P., De Vicente, J., Marchal, J. A., and Boulaiz, H. (2019). Clinical trials of thermosensitive nanomaterials: an overview. Nanomaterials 9:191. doi: 10.3390/nano9020191

Pedrosa, P., Vinhas, R., Fernandes, A., and Baptista, P. V. (2015). Gold nanotheranostics: proof-of-concept or clinical tool? Nanomaterials 5, 1853-1879. doi: 10.3390/nano5041853

Qiao, C., Yang, J., Shen, Q., Liu, R., Li, Y., Shi, Y., et al. (2018). Traceable nanoparticles with dual targeting and ROS response for RNAi-based immunochemotherapy of intracranial glioblastoma treatment. Adv. Mater. 30:1705054. doi: 10.1002/adma.201705054

Ren, Q., Liang, Z., Jiang, X., Gong, P., Zhou, L., Sun, Z., et al. (2019). Enzyme and $\mathrm{pH}$ dual-responsive hyaluronic acid nanoparticles mediated combination of photodynamic therapy and chemotherapy. Int. J. Biol. Macromol. 130, 845-852. doi: 10.1016/j.ijbiomac.2019.03.030

Shamsi, M., Saghafian, M., Dejam, M., and Sanati-Nezhad, A. (2018). Mathematical modeling of the function of warburg effect in tumor microenvironment. Sci. Rep. 8:8903. doi: 10.1038/s41598-018-27303-6

Silva, C. O., Pinho, J. O., Lopes, J. M., Almeida, A. J., Gaspar, M. M., and Reis, C. (2019). Current trends in cancer nanotheranostics: metallic, polymeric, and lipid-based systems. Pharmaceutics 11:22. doi: 10.3390/pharmaceutics11010022

Sneider, A., Vandyke, D., Paliwal, S., and Rai, P. (2017). Remotely triggered nano-theranostics for cancer applications. Nanotheranostics 1, 1-22. doi: $10.7150 /$ ntno. 17109

Song, X. R., Li, S. H., Dai, J., Song, L., Huang, G., Lin, R., et al. (2017). Polyphenolinspired facile construction of smart assemblies for ATP-and $\mathrm{pH}$-responsive tumor MR/optical imaging and photothermal therapy. Small 13:1603997. doi: 10.1002/smll.201603997

Sun, C.-Y., Cao, Z., Zhang, X.-J., Sun, R., Yu, C.-S., and Yang, X. (2018). Cascade-amplifying synergistic effects of chemo-photodynamic therapy using ROS-responsive polymeric nanocarriers. Theranostics 8, 2939-2953. doi: $10.7150 /$ thno. 24015

Tan, J., Deng, Z., Liu, G., Hu, J., and Liu, S. (2018). Anti-inflammatory polymersomes of redox-responsive polyprodrug amphiphiles with inflammation-triggered indomethacin release characteristics. Biomaterials 178, 608-619. doi: 10.1016/j.biomaterials.2018.03.035

Tekade, R. K., and Sun, X. (2017). The Warburg effect and glucosederived cancer theranostics. Drug Discov. Today 22, 1637-1653. doi: 10.1016/j.drudis.2017.08.003

Trachootham, D., Alexandre, J., and Huang, P. (2009). Targeting cancer cells by ROS-mediated mechanisms: a radical therapeutic approach? Nat. Rev. Drug Discov. 8, 579-591. doi: 10.1038/nrd2803

Ushitora, M., Sakurai, F., Yamaguchi, T., Nakamura, S.-I., Kondoh, M., Yagi, K., et al. (2010). Prevention of hepatic ischemia-reperfusion injury by preadministration of catalase-expressing adenovirus vectors. J. Control. Release 142, 431-437. doi: 10.1016/j.jconrel.2009.11.024 
Vidallon, M. L. P., Douek, A. M., Quek, A., Mcliesh, H., Kaslin, J., Tabor, R. F., et al. (2020). Gas-generating, pH-responsive calcium carbonate hybrid particles with biomimetic coating for contrast-enhanced ultrasound imaging. Part. Part. Syst. Char. 37:1900471. doi: 10.1002/ppsc.201900471

Wang, J., Macewan, S. R., and Chilkoti, A. (2017a). Quantitative mapping of the spatial distribution of nanoparticles in endo-lysosomes by local pH. Nano Lett. 17, 1226-1232. doi: 10.1021/acs.nanolett.6b05041

Wang, J., Tao, W., Chen, X., Farokhzad, O. C., and Liu, G. (2017b). Emerging advances in nanotheranostics with intelligent bioresponsive systems. Theranostics 7, 3915-3919. doi: 10.7150/thno.21317

Wong, X. Y., Sena-Torralba, A., Alvarez-Diduk, R., Muthoosamy, K., and Merkoçi, A. (2020). Nanomaterials for nanotheranostics: tuning their properties according to disease needs. ACS Nano 14, 2585-2627. doi: 10.1021 /acsnano.9b08133

Wu, X., Sun, X., Guo, Z., Tang, J., Shen, Y., James, T. D., et al. (2014). In vivo and in situ tracking cancer chemotherapy by highly photostable NIR fluorescent theranostic prodrug. J. Am. Chem. Soc. 136, 3579-3588. doi: 10.1021/ja412380j

Xiao, J., Zhang, G., Xu, R., Chen, H., Wang, H., Tian, G., et al. (2019). A pH-responsive platform combining chemodynamic therapy with limotherapy for simultaneous bioimaging and synergistic cancer therapy. Biomaterials 216:119254. doi: 10.1016/j.biomaterials.2019.119254

Xiao, M., Sun, W., Fan, J., Cao, J., Li, Y., Shao, K., et al. (2018). Aminopeptidase-Nactivated theranostic prodrug for NIR tracking of local tumor chemotherapy. Adv. Func. Mater. 28:1805128. doi: 10.1002/adfm.201805128

Yan, X., Niu, G., Lin, J., Jin, A. J., Hu, H., Tang, Y., et al. (2015). Enhanced fluorescence imaging guided photodynamic therapy of sinoporphyrin sodium loaded graphene oxide. Biomaterials 42, 94-102. doi: 10.1016/j.biomaterials.2014.11.040

Yang, G., Sun, X., Liu, J., Feng, L., and Liu, Z. (2016a). Light-responsive, singlet-oxygen-triggered on-demand drug release from photosensitizer-doped mesoporous silica nanorods for cancer combination therapy. Adv. Func. Mater. 26, 4722-4732. doi: 10.1002/adfm.201600722

Yang, K., Feng, L., and Liu, Z. (2016b). Stimuli responsive drug delivery systems based on nano-graphene for cancer therapy. Adv. Drug Deliv. Rev. 105, 228-241. doi: 10.1016/j.addr.2016.05.015

Yang, X., Du, H., Liu, J., and Zhai, G. (2015). Advanced nanocarriers based on heparin and its derivatives for cancer management. Biomacromolecules 16, 423-436. doi: 10.1021/bm501532e

Yang, X., Shi, X., Ji, J., and Zhai, G. (2018a). Development of redoxresponsive theranostic nanoparticles for near-infrared fluorescence imagingguided photodynamic/chemotherapy of tumor. Drug Deliv. 25, 780-796. doi: 10.1080/10717544.2018.1451571

Yang, Z., Dai, Y., Yin, C., Fan, Q., Zhang, W., Song, J., et al. (2018b). Activatable semiconducting theranostics: simultaneous generation and ratiometric photoacoustic imaging of reactive oxygen species in vivo. Adv. Mater. 30:1707509. doi: 10.1002/adma.201707509
Yu, C.-J., Wu, S.-M., and Tseng, W.-L. (2013). Magnetite nanoparticle-induced fluorescence quenching of adenosine triphosphate-BODIPY conjugates: application to adenosine triphosphate and pyrophosphate sensing. Anal. Chem. 85, 8559-8565. doi: 10.1021/ac400919j

Yu, G., Yung, B. C., Zhou, Z., Mao, Z., and Chen, X. (2018). Artificial molecular machines in nanotheranostics. ACS Nano 12, 7-12. doi: 10.1021/acsnano.7b07851

Yue, C., Yang, Y., Zhang, C., Alfranca, G., Cheng, S., Ma, L., et al. (2016). ROS-responsive mitochondria-targeting blended nanoparticles: chemo-and photodynamic synergistic therapy for lung cancer with on-demand drug release upon irradiation with a single light source. Theranostics 6, 2352-2366. doi: 10.7150/thno.15433

Yun, J., Mullarky, E., Lu, C., Bosch, K. N., Kavalier, A., Rivera, K., et al. (2015). Vitamin C selectively kills KRAS and BRAF mutant colorectal cancer cells by targeting GAPDH. Science 350, 1391-1396. doi: 10.1126/science.aaa5004

Zhang, P., Hu, C., Ran, W., Meng, J., Yin, Q., and Li, Y. (2016). Recent progress in light-triggered nanotheranostics for cancer treatment. Theranostics 6, 948-968. doi: $10.7150 /$ thno. 15217

Zhao, X., Li, Y., Jin, D., Xing, Y., Yan, X., and Chen, L. (2015). A near-infrared multifunctional fluorescent probe with an inherent tumor-targeting property for bioimaging. Chem. Commun. 51, 11721-11724. doi: 10.1039/C5CC03878B

Zhao, X., Yang, C.-X., Chen, L.-G., and Yan, X.-P. (2017). Dual-stimuli responsive and reversibly activatable theranostic nanoprobe for precision tumor-targeting and fluorescence-guided photothermal therapy. Nat. Commun. 8:14998. doi: $10.1038 /$ ncomms 14998

Zhou, F., Feng, B., Wang, T., Wang, D., Cui, Z., Wang, S., et al. (2017). Theranostic prodrug vesicles for reactive oxygen species-triggered ultrafast drug release and local-regional therapy of metastatic triple-negative breast cancer. Adv. Func. Mater. 27:1703674. doi: 10.1002/adfm.201703674

Zhu, J., Wang, G., Alves, C. S., TomáS., H., Xiong, Z., et al. (2018). Multifunctional dendrimer-entrapped gold nanoparticles conjugated with doxorubicin for $\mathrm{pH}$-responsive drug delivery and targeted computed tomography imaging. Langmuir 34, 12428-12435. doi: 10.1021/acs.langmuir.8b0 2901

Conflict of Interest: The authors declare that the research was conducted in the absence of any commercial or financial relationships that could be construed as a potential conflict of interest.

Copyright (C) 2020 Parodi, Rudzinska, Leporatti, Anissimov and Zamyatnin. This is an open-access article distributed under the terms of the Creative Commons Attribution License (CC BY). The use, distribution or reproduction in other forums is permitted, provided the original author(s) and the copyright owner(s) are credited and that the original publication in this journal is cited, in accordance with accepted academic practice. No use, distribution or reproduction is permitted which does not comply with these terms. 